\title{
ARTí́CULO
}

\section{Calidad de los sedimentos marinos entre el Estrecho de Magallanes y el Cabo de Hornos (Patagonia chilena) en función de pruebas de toxicidad}

\author{
Marine sediment quality between Estrecho de Magallanes and Cabo de Hornos \\ (Chilean Patagonia), according to toxicity tests
}

\author{
Anny Rudolph ${ }^{1}$, Vanessa Novoa ${ }^{2}$, Ramón Ahumada ${ }^{1}, K$ atia Saez $^{3}$ y Nelson Silva ${ }^{4}$ \\ ${ }^{1}$ Facultad de Ciencias, Universidad Católica de la Santísima Concepción, Caupolicán 491, Concepción, Chile. annyr@ucsc.cl \\ ${ }^{2}$ Programa de Postgrado Centro EULA, Universidad de Concepción, Víctor Lamas 1290, Concepción, Chile \\ ${ }^{3}$ Departamento de Matemáticas y Estadística, Universidad de Concepción, Víctor Lamas 1290, Concepción, Chile \\ ${ }^{4}$ Escuela de Ciencias del Mar, Pontificia Universidad Católica de Valparaíso, Avenida Brasil 2950, Valparaíso, Chile
}

\begin{abstract}
The southern Chilean Patagonia is being incorporated into the productive activity through their water bodies applied in aquaculture. This makes it necessary to establish guidelines for sediment quality. The aim of this study was to analyze the environmental quality of sediments collected during an oceanographic cruise from Estrecho de Magallanes $\left(52^{\circ} 41.1^{\prime} \mathrm{S}\right)$ to Cabo de Hornos $\left(55^{\circ} \mathrm{O} .9^{\prime} \mathrm{S}\right)$, through testing of survival, fertilization and growth. The testing organisms were Tisbe longicornis a benthic arthropoda, Arbacia spatuligera an echinoderm and 3 microalgae: Dunaliella tertiolecta, $D$. salina and Isochrysis galbana. The principal component analysis (PCA) and dendrogram allowed verify in the first place the sensitivity of the assays applied. Furthermore, PCA allowed observing clusters of stations based on the growth of the 3 microalgae, showing that the first 2 components accounted for almost $80 \%$ of the total variance. The stations that behaved differently from the control groups were two in the southern sector and one in the northwestern sector, due to their oceanic influence. These result showed that the slight difference between fertilization trials, survival and growth of microalgae on the controls, allow us to suggest that this area is in pristine condition even from the standpoint of sediment quality.
\end{abstract}

Key words: Ecotoxicology, pristine regions, environmental quality

Resumen.- La zona sur de la Patagonia chilena se está incorporando a la actividad productiva, a través del uso de sus cuerpos de agua en la acuicultura, lo que hace necesario establecer líneas bases de calidad de sus sedimentos. El objetivo de este trabajo fue analizar la calidad de los sedimentos recolectados en el sector entre el Estrecho Magallanes $\left(52^{\circ} 41,1^{\prime}\right)$ y Cabo de Hornos $\left(55^{\circ} 0,9^{\prime} \mathrm{S}\right)$, a través de ensayos de supervivencia, fecundación y crecimiento. Los organismos utilizados fueron un artrópodo bentónico, Tisbe longicornis; gametos de un equinodermo, Arbacia spatuligera y 3 microalgas, Dunaliella tertiolecta, D. salina e Isochrysis galbana. El análisis de componentes principales y dendograma confirmó la sensibilidad de los ensayos utilizados y permitió observar agrupaciones de estaciones sobre la base del crecimiento de las 3 microalgas, observándose que las primeras componentes representaron casi un $80 \%$ de la varianza total. Las estaciones que se comportaron diferentes de los grupos control fueron dos en el sector sur y una en el sector noroeste, debido a su mayor influencia oceánica. Se concluye que la escasa diferencia entre los ensayos de fecundación, supervivencia y crecimiento de las microalgas respecto de los controles, permite considerar a esta área, como prístina desde el punto de vista de la calidad del sedimento.

Palabras clave: Ecotoxicología, regiones prístinas, calidad ambiental 


\section{INTRODUCCIÓN}

La costa de la Patagonia chilena que se extiende desde los $42^{\circ} 50^{\prime}$ hasta los $56^{\circ} 30^{\prime}$ 's, se caracteriza por una intrincada red de canales, fiordos, senos y golfos, los que se formaron durante el pleistoceno por el socavamiento producido por el avance y retroceso de los glaciares y el hundimiento tectónico del valle central de Chile (Borgel 1970-1971). Al subir el nivel del mar durante el holoceno, producto del retroceso de la era glaciar, esta zona fue inundada por las aguas del mar adyacente, el aporte de ríos y el derretimiento de glaciares. Debido a sus características topográficas, la zona estuarina patagónica chilena puede ser sectorizada en 3 zonas mayores, una norpatagónica, entre Puerto Montt y península de Taitao, una patagónica central, entre península de Taitao y el Estrecho de Magallanes y una sur patagónica, entre el Estrecho de Magallanes y el Cabo de Hornos (Pickard 1971, Silva \& Palma 2008). De ellas, la más estudiada a la fecha corresponde a la norte, la cual es utilizada intensamente para el cultivo de especies marinas.

En la zona norpatagónica el análisis de calidad realizado en los sedimentos recolectados en el sector central de sus fiordos y canales, utilizando ensayos de toxicidad con microalgas, mostraron incrementos significativos en la densidad celular respecto de los controles, atribuible a desbalances en el contenido de fósforo y/o nitrógeno de los sedimentos (Rudolph et al. 2007). Sin embargo, muestras recolectadas en la misma área, pero en sectores costeros cercanos a los centros de cultivo, presentaron sedimentos alterados, en su mayoría anóxicos en que el comportamiento de los organismos en los ensayos fue significativamente diferente al de los controles (Rudolph et al. 2009, 2010).

La zona sur patagónica es la menos conocida, debido en parte a su difícil acceso, su intrincada e irregular topografía y un clima hostil, que le ha permitido permanecer protegida del impacto de las actividades humanas (Rozzi et al. 2007, Mansilla et al. 2011). Presenta extensos canales que conectan el Océano Pacífico con el Océano Atlántico: el Estrecho de Magallanes y la sección canal Beagle-brazo Sudoeste, los que se suman a la intrincada y extensa red de canales, fiordos, senos y bahías, de características topográficas y oceanográficas diversas (Pickard 1971, Valdenegro \& Silva 2003, Sobarzo 2009). La presencia de constricciones-umbrales en esta zona, da origen a la formación de una serie de micro cuencas profundas (300-1.300 m), que interrumpen la continuidad de la circulación estuarina entre el océano abierto y los canales interiores (Antezana 1999,
Valdenegro \& Silva 2003). La existencia de estas constricciones y micro cuencas, además de sus diferencias biológicas, generan diferentes dinámicas de circulación y de sedimentación de los lixiviados continentales, lo que plantea interrogantes sobre el efecto de eventuales perturbaciones antropogénicas (Antezana et al. 2002).

Oceanográficamente, la columna de agua del ecosistema de aguas interiores de la Provincia Magallánica se caracteriza por una estructura de 2 capas separadas por una fuerte haloclina, con circulación estuarina positiva, generada por el ingreso de agua dulce continental (lluvia, ríos y deshielos) y de agua de mar. La capa superficial muestra salinidades que fluctúan entre 26 y 31 y la capa profunda, salinidades que fluctúan entre 31 y 34, y relativamente altos contenidos de oxígeno disuelto e.g., sobre $4 \mathrm{ml} \mathrm{L}^{-1}$ (Valdenegro \& Silva 2003, Sievers \& Silva 2008). Desde el punto de vista de la composición química del sedimento y en función del contenido de carbono inorgánico, Silva \& Ortiz (2002) identifican 3 zonas; 2 con contenidos de carbono inorgánico entre 4 y 11\% (e.g., estaciones del extremo occidental del Estrecho de Magallanes y porción oriental del canal Beagle y Bahía Nassau) y una tercera zona con bajos contenidos de carbono inorgánico, entre 0 y $2 \%$, ubicada en el centro del área. Además, la relación carbono orgánico: nitrógeno orgánico (C:N) en el sedimento, permite reconocer 2 zonas: una donde el origen del sedimento se asocia a características químicas propias de un sedimento marino y otra zona, cuyos sedimentos se asocian a la presencia de sedimentos de origen marino, con cierto grado de descomposición diagenética de su materia orgánica, con aporte de material edáfico y/o terrígeno, el que habría sido arrastrado por ríos o escurrimiento costero. En cuanto al contenido de metales traza en los sedimentos, se observan concentraciones en ( $\mu \mathrm{g} \mathrm{g}^{-1}$ de sedimento) que son características de la línea base de los fiordos australes chilenos, e.g., Cd 0,3 $\pm 0,1$ $\left(\mu \mathrm{g} \mathrm{g}^{-1}\right)$; Co 13,2 $\pm 2,4\left(\mu \mathrm{g} \mathrm{g}^{-1}\right)$; Cr 60,4 $\pm 12,1\left(\mu \mathrm{g} \mathrm{g}^{-1}\right)$; $\mathrm{Cu}$ 21,9 \pm 7,8 $\left(\mu \mathrm{g} \mathrm{g}^{-1}\right) ; \mathrm{Ni} 29,7 \pm 7,8\left(\mu \mathrm{g} \mathrm{g}^{-1}\right) ; \mathrm{Pb} 22,6 \pm 5,1(\mu \mathrm{g}$ $\left.\mathrm{g}^{-1}\right)$; Sr 266,5 \pm 94,5 $\left(\mu \mathrm{g} \mathrm{g}^{-1}\right) ; \mathrm{V} 146,4 \pm 18,2\left(\mu \mathrm{g} \mathrm{g}^{-1}\right)$ y Zn $112,4 \pm 21,6\left(\mu \mathrm{g} \mathrm{g}^{-1}\right)$ (Ahumada et al. 2002). El único elemento que presentaría una concentración relativamente mayor en el área es el Ba con $549 \pm 121\left(\mu \mathrm{g} \mathrm{g}^{-1}\right)$ (Ahumada et al. 2002).

En esta zona, la composición química del sedimento está influida tanto por los aportes oceánicos como continentales. Los sedimentos de la zona no registran alteraciones antropogénicas, ya que los elementos 
químicos presentan concentraciones y distribución dentro de rangos naturales, originados principalmente por lixiviación continental (Ahumada et al. 2002). No obstante, esta región está siendo incorporada a la explotación de recursos naturales e introducción de actividades de la salmonicultura, por lo que el objetivo de este trabajo fue evaluar la calidad de los sedimentos del área del sector del Estrecho Magallanes a Cabo de Hornos $\left(52^{\circ} 41,1^{\prime} y 55^{\circ} 0,9^{\prime} \mathrm{S}\right)$ a través de ensayos de toxicidad utilizando pruebas de supervivencia, fecundación y crecimiento.

\section{Materiales y MÉTODOS}

El muestreo de sedimentos del área comprendida entre el Estrecho de Magallanes y Cabo de Hornos, fue realizado entre el 31 de octubre y 12 de noviembre de 2010, durante el crucero oceanográfico CIMAR (Campaña de Investigación Marina en Áreas Remotas) 16 Fiordo, a bordo del buque 'AGOR Vidal Gormaz', coordinado por el Comité Oceanográfico Nacional (CONA, Chile). Las muestras fueron recolectadas a profundidades que fluctuaron entre 97 y 520 m, en 18 estaciones distribuidas en la zona de muestreo (Fig.1). Las muestras fueron recolectadas en triplicado desde los primeros $5 \mathrm{~cm}$ (aproximadamente $250 \mathrm{~g}$ cada una), las cuales fueron guardadas congeladas $\left(-4^{\circ} \mathrm{C}\right)$, por un periodo no mayor de 3 meses, que duró el muestreo, traslado y análisis. En el laboratorio se subdividió cada réplica con el objeto de realizar análisis de materia orgánica, como pérdida de peso por ignición (Luczak et al. 1997) y los análisis de toxicidad.

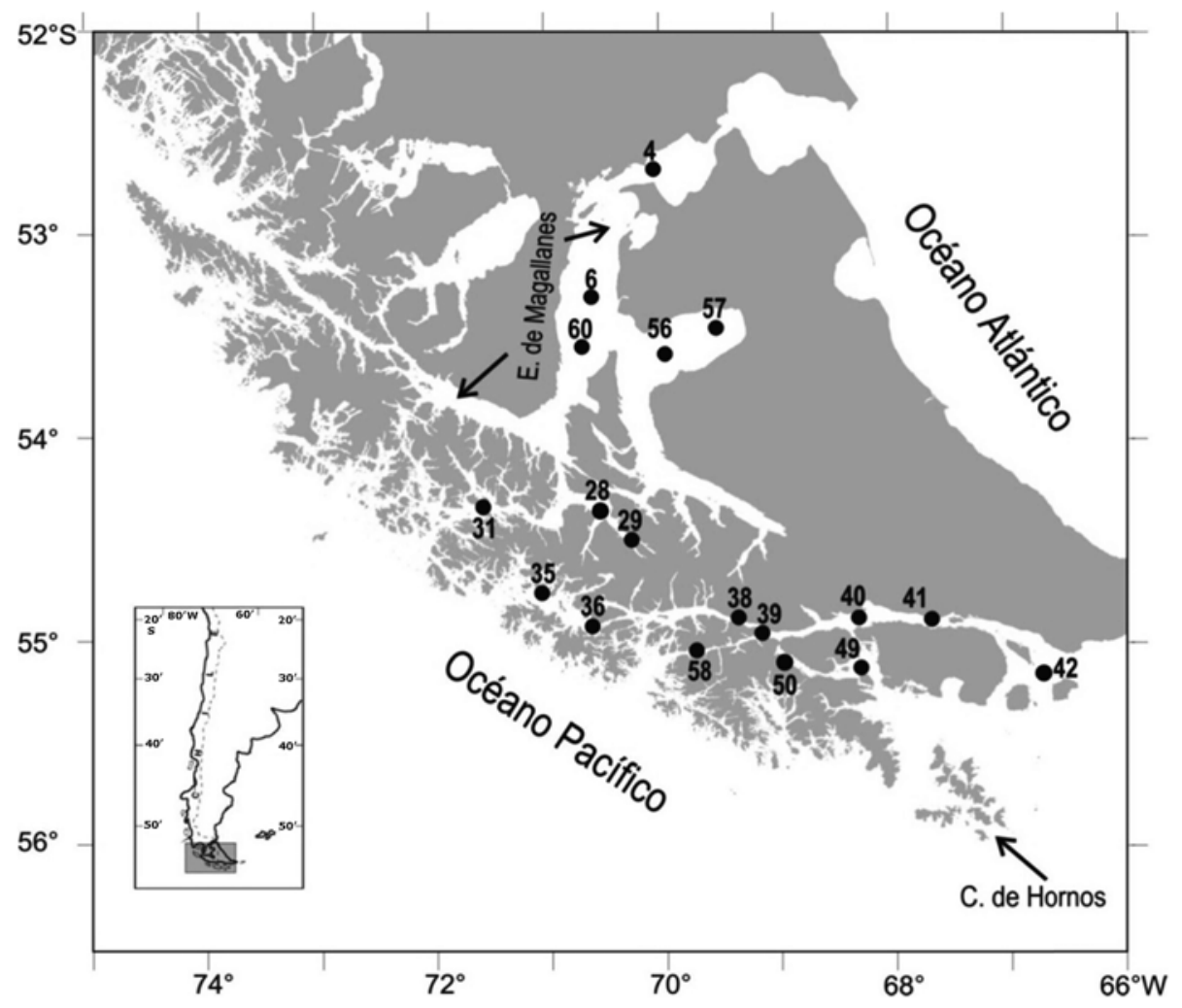

Figura 1. Ubicación de las estaciones muestreadas durante el crucero oceanográfico CIMAR 16 Fiordo, octubre-noviembre $\mathbf{2 0 1 0}$ / Location of stations sampled during the oceanographic cruise CIMAR 16 Fjord, October-November 2010 
El análisis de calidad del sedimento se realizó mediante una batería de ensayos compuesta por pruebas de supervivencia, con el copépodo harpacticoide Tisbe longicornis (George, 1993), pruebas de fecundación, con gametos del equinodermo Arbacia spatuligera (Valenciennes, 1841) y test de densidad celular (cel. $\mathrm{ml}^{-1}$ ), con 3 especies de microalgas Isochrysis galbana (Parke), Dunaliella tertiolecta (Butcher, 1959) y D. salina (Dunal).

Las pruebas seleccionadas se realizaron exponiendo los organismos blancos a un elutriado de los sedimentos. El elutriado de cada muestra fue obtenido según la metodología de Dinnel \& Strober (1985), agitando 150 g de sedimento con $150 \mathrm{~mL}$ de agua de mar filtrada y aireada, en un Heidolph ${ }^{\circledR}$ Unimax 2010 a 5 rpm por $10 \mathrm{~min}$, para posteriormente dejar en frío $\left(4^{\circ} \mathrm{C}\right)$ por $12 \mathrm{~h}$ con el objeto de separar la fase líquida (elutriado) de los sedimentos. Los organismos para el estudio i.e., T. longicornis y A. spatuligera fueron recolectados en un área de baja alteración en la Bahía Coliumo (3650’S; 7255‘W) y las microalgas $D$. tertiolecta, $D$. salina e I. galbana fueron adquiridas en el laboratorio de cultivos de microalgas de la Universidad de Concepción.

\section{Prueba de supervivencia con T. Longicornis}

El ensayo se realizó según las especificaciones de Larraín et al. (1998). Hembras ovígeras adultas fueron recolectadas con un tamiz de $250 \mu \mathrm{m}$ y cultivadas aisladas en pequeños acuarios de vidrio, hasta la eclosión de los huevos, con aireación constante y alimentación en base a un cultivo mixto de microalgas. El ensayo se condujo con organismos juveniles de una misma cohorte de ca.15 días de vida (i.e., 0,6 mm de longitud). Se utilizó elutriado preparado con el sedimento bajo prueba. Los resultados se contrastaron con controles negativos. Los ensayos de sensibilidad se condujeron con soluciones de $\mathrm{K}_{2} \mathrm{Cr}_{2} \mathrm{O}_{7 \text { (p.a) }}$ entre 1 y $50 \mathrm{mgL}^{-1}$. Los ensayos con los sedimentos, de sensibilidad y control, se condujeron por $48 \mathrm{~h}$ en forma paralela, con 5 individuos por cubeta y en triplicado.

\section{Prueba de fertilización con A. SPATUligera}

El ensayo se realizó según las especificaciones indicadas en USEPA (1988) y las modificaciones introducidas por Zúñiga (1999). Básicamente consiste en producir una fecundación artificial de los óvulos del erizo $A$. spatuligera en presencia de un elutriado preparado con el sedimento bajo prueba. Los resultados se contrastaron con controles negativos. El análisis de sensibilidad se condujo con soluciones de $\mathrm{CuSO}_{4 \text { (p.a) }}$ entre 3,1 y $100 \mu \mathrm{g} \mathrm{L}^{-1}$. Los ensayos de sensibilidad y blanco, se realizaron en forma paralela por un periodo de $60 \mathrm{~min}$, utilizando soluciones de 7 × $10^{7}$ espermios $\mathrm{mL}^{-1}$ y 2000 óvulos $\mathrm{mL}^{-1}$, en cuadruplicado.

Prueba de Crecimiento con I. galbana, D. salina y D. TERTIOLECTA

Los ensayos se condujeron según la metodología propuesta por la U.S. Environmental Protection Agency (USEPA 1978) y consideró las modificaciones introducidas por Cifuentes et al. (1998) y NCH 2706 (2002) $)^{1}$. Las microalgas fueron cultivadas con el medio de cultivo Guillar a $17^{\circ} \mathrm{C} \pm 1$ y luz PAR continua de 28.000 lux. $D$. salina fue cultivada en un medio salino, con salinidad semejante a la del agua de mar. Se utilizó $10 \mathrm{~mL}$ del elutriado preparado con el sedimento bajo prueba e inóculos de cultivo inicial. Los resultados se contrastaron con controles negativos. El análisis de sensibilidad se realizó con concentraciones entre 0 y $100 \mathrm{mgL}^{-1}$ de $\mathrm{K}_{2} \mathrm{Cr}_{2} \mathrm{O}_{7 \text { (p.a) }}$. Los ensayos con los sedimentos, de sensibilidad y control, para cada una de las especies de algas, se realizaron en forma paralela y en cuadruplicado. La densidad celular (cel. $\mathrm{mL}^{-1}$ ) fue determinada mediante recuento de células con cámara de Neubauer a tiempo cero (To) y a 96 h (Tf).

\section{AnÁlisis ESTADÍstico}

Los diferentes índices de los ensayos de sensibilidad i.e., $\mathrm{CL}_{50}$ (concentración letal para el 50\% de los organismos sometidos al ensayo) para T. longicornis; $\mathrm{CE}_{50}$ (concentración efectiva en el 50\% de los organismos sometidos al ensayo) en el ensayo de A. spatuligera y el $\mathrm{CI}_{50}$ (concentración que inhibió al $50 \%$ el crecimiento celular de las microalgas (cel. $\mathrm{mL}^{-1}$ ) en I. galbana, D. salina y D. tertiolecta, fueron calculados mediante análisis probit (EPA Probit Análisis Program, versión 1,4, Finney 1971). Para analizar la normalidad de los datos se aplicó el test de Shapiro-Wilk y para observar la homogeneidad de la varianza se utilizó el test de Cochrane. Los resultados de cada ensayo fueron comparados con su respectivo grupo control, a través de una prueba de ANDEVA de una vía.

${ }^{1} \mathrm{NCH}$ 2706. 2002. Calidad de agua - bioensayo de inhibición de crecimiento de algas en agua dulce con Selenastrum capricornutum (Raphidocelis subcapitata), 28 pp. Instituto Chileno de Normalización, Santiago. 
Además, se realizó análisis de componentes principales para estudiar la respuesta de las microalgas de acuerdo a su crecimiento, en las distintas estaciones y de conglomerados y las posibles semejanzas y/o diferencias espaciales, a una distancia de 3 unidades euclidianas. Para realizar estos análisis estadísticos se utilizó el programa computacional STATISTICA versión 6.0 (StatSoft. Inc. 2001) ${ }^{2}$.

\section{Resultados}

\section{MATERIA ORgáNICA}

Las muestras, independiente de las diferencia en profundidad en que fueron tomadas, mostraron contenidos de materia orgánica total cercanos al 5\% en el $67 \%$ de las estaciones. Contenidos cercanos al $11 \%$ se observó en las estaciones interiores 58 y 49, y bajo el 4\% en las estaciones 29, 38 y 39 (Tabla 1).

Tabla 1. Ubicación, profundidad y contenidos de materia orgánica total (MOT) de las estaciones de muestreo. CIMAR 16 Fiordo, octubre-noviembre $\mathbf{2 0 1 0}$ / Location, depth and organic matter content of the sampling stations. CIMAR 16 Fjord, OctoberNovember 2010

\begin{tabular}{cllrr}
\hline Estación & Lat. S & Long. O & Z (m) & \multicolumn{1}{c}{ MOT (\%) } \\
\hline 4 & $52^{\circ} 40,9^{\prime}$ & $70^{\circ} 09^{\prime}$ & 44 & $5,28 \pm 0,41$ \\
6 & $53^{\circ} 16,5^{\prime}$ & $70^{\circ} 41,2^{\prime}$ & 196 & $6,34 \pm 0,22$ \\
60 & $53^{\circ} 34,66^{\prime}$, & $70^{\circ} 35,99^{\prime}$ & 268 & $4,46 \pm 0,07$ \\
28 & $54^{\circ} 20,2^{\prime}$ & $70^{\circ} 33,6^{\prime}$, & 259 & $5,75 \pm 0,15$ \\
29 & $54^{\circ} 29,7^{\prime}$ & $70^{\circ} 19,0^{\prime}$ & 229 & $3,51 \pm 0,71$ \\
31 & $54^{\circ} 20,7^{\prime}$ & $71^{\circ} 37,6^{\prime}$ & 429 & $5,83 \pm 0,41$ \\
35 & $54^{\circ} 46,5^{\prime}$ & $71^{\circ} 07,5^{\prime}$ & 407 & $5,59 \pm 0,14$ \\
36 & $54^{\circ} 55,8^{\prime}$ & $71^{\circ} 42,8^{\prime}$ & 97 & $8,00 \pm 0,37$ \\
38 & $54^{\circ} 53,0^{\prime}$ & $69^{\circ} 28,1^{\prime}$ & 300 & $2,72 \pm 0,05$ \\
39 & $54^{\circ} 58,2^{\prime}$ & $69^{\circ} 02,2^{\prime}$ & 195 & $3,37 \pm 0,12$ \\
40 & $54^{\circ} 54,2^{\prime}$ & $68^{\circ} 28,1^{\prime}$ & 193 & $6,73 \pm 0,12$ \\
41 & $54^{\circ} 54,3^{\prime}$ & $67^{\circ} 43,5^{\prime}$ & 173 & $6,7 \pm 1,65$ \\
42 & $55^{\circ} 00,9^{\prime}$ & $66^{\circ} 47,5^{\prime}$ & 111 & $6,75 \pm 0,71$ \\
49 & $55^{\circ} 08,0^{\prime}$ & $68^{\circ} 13,0^{\prime}$ & 389 & $11,30 \pm 0,98$ \\
50 & $55^{\circ} 08,2^{\prime}$ & $68^{\circ} 49,0^{\prime}$ & 107 & $8,78 \pm 1,20$ \\
58 & $55^{\circ} 04,38^{\prime}$ & $69^{\circ} 48,66^{\prime}$ & 520 & $11,02 \pm 0,97$ \\
57 & $53^{\circ} 27,9^{\prime}$ & $69^{\circ} 30,2^{\prime}$ & 200 & $6,97 \pm 0,12$ \\
56 & $53^{\circ} 32,8^{\prime}$ & $69^{\circ} 51,5^{\prime}$ & 44 & $5,03 \pm 0,18$ \\
\hline & & &
\end{tabular}

\section{SUPERVIVENCIA T. LONGICORNIS}

El análisis de sensibilidad de los juveniles de la cohorte utilizada arrojó un $\mathrm{LC}_{50}$ estimado de $51,1 \mathrm{mg} \mathrm{L}^{-1}$ al $95 \%$ de confianza, para soluciones de $\mathrm{K}_{2} \mathrm{Cr}_{2} \mathrm{O}_{7}$. Los controles negativos presentaron un promedio de supervivencia de $100 \%$. No se observó diferencias significativas en la supervivencia de los T. longicornis sometidos a los ensayos de los sedimentos en las estaciones analizadas y el grupo control $(\mathrm{KW}-\mathrm{H}(18 / 57)=23,89 ; P=0,1586)$ excepto en la estación 31 (Fig. 2).

\section{Prueba de fecundación con A. SPATUligera}

$\mathrm{El}$ análisis de sensibilidad de los gametos con soluciones de $\mathrm{CuSO}_{4}$ arrojó un $\mathrm{EC}_{50}$ estimado de $14,1 \mu \mathrm{gL}^{-1}$ al $95 \%$ de confianza. Los controles negativos presentaron un promedio de fecundación de $100 \%$. Se encontró diferencias significativas en la fecundación de los gametos de A. spatuligera expuestos a los sedimentos de las estaciones respecto al blanco. Los porcentajes de fecundación fueron significativamente menores $(\mathrm{KW}-\mathrm{H}$ $(18 / 57)=51,892 ; P=0,0001)$ en los ensayos con los sedimentos de las estaciones $31,36,39,40,41,42,49,50$, 56, 57 y 58, respecto del grupo control (Fig. 3).

\section{Prueba de Crecimiento con D. tertiolecta, D. Salina Y I. GALBANA (CEL ML $\left.{ }^{-1} 10^{4}\right)$}

$\mathrm{El}$ análisis de sensibilidad de las microalgas con soluciones de $\mathrm{K}_{2} \mathrm{Cr}_{2} \mathrm{O}_{7}$ arrojó un $\mathrm{IC}_{50}$ estimado de $72 \mathrm{mg} \mathrm{L}^{-1}, 65 \mathrm{mg} \mathrm{L}^{-1}$, $55 \mathrm{mg} \mathrm{L}^{-1}$ al 95\% de confianza, respectivamente. El análisis del crecimiento mostró diferencias significativas al término del ensayo (i.e., 96 h) en la densidad celular de las 3 microalgas, en relación a sus grupos control (Fig. 4). I. galbana presentó diferencias significativas en la densidad celular de las muestras respecto del grupo control (ANDEVA, $\mathrm{F}_{(18,56)}=9,73 ; P=0,0001$ ), en las estaciones 5, 6, 28, 29, 31, 35, 36, 38, 39, 40, 42, 58 y 60, según la prueba a posteriori de Tukey. D. salina también presentó diferencias significativas en la densidad celular en las muestras respecto del grupo control (ANDEVA, $\left.\mathrm{F}_{(18,56)}=27,64 ; P=0,0001\right)$, en las estaciones $41,42,49,50$ y 56, según la prueba a posteriori de Tukey. $D$. tertiolecta presentó diferencias significativas en la densidad celular de las muestras respecto del grupo control (ANDEVA, $\left.\mathrm{F}_{(18,56)}=11,04 ; P=0,0001\right)$, en las muestras de las estaciones $5,28,29,31,35,36,39,40,41$ y 57, según la prueba $a$ posteriori de Tukey (Fig. 4).

${ }^{2}$ StatSoft. Inc. (2001). Statistica (data analysis software system). <www.statsoft.com $>$ 


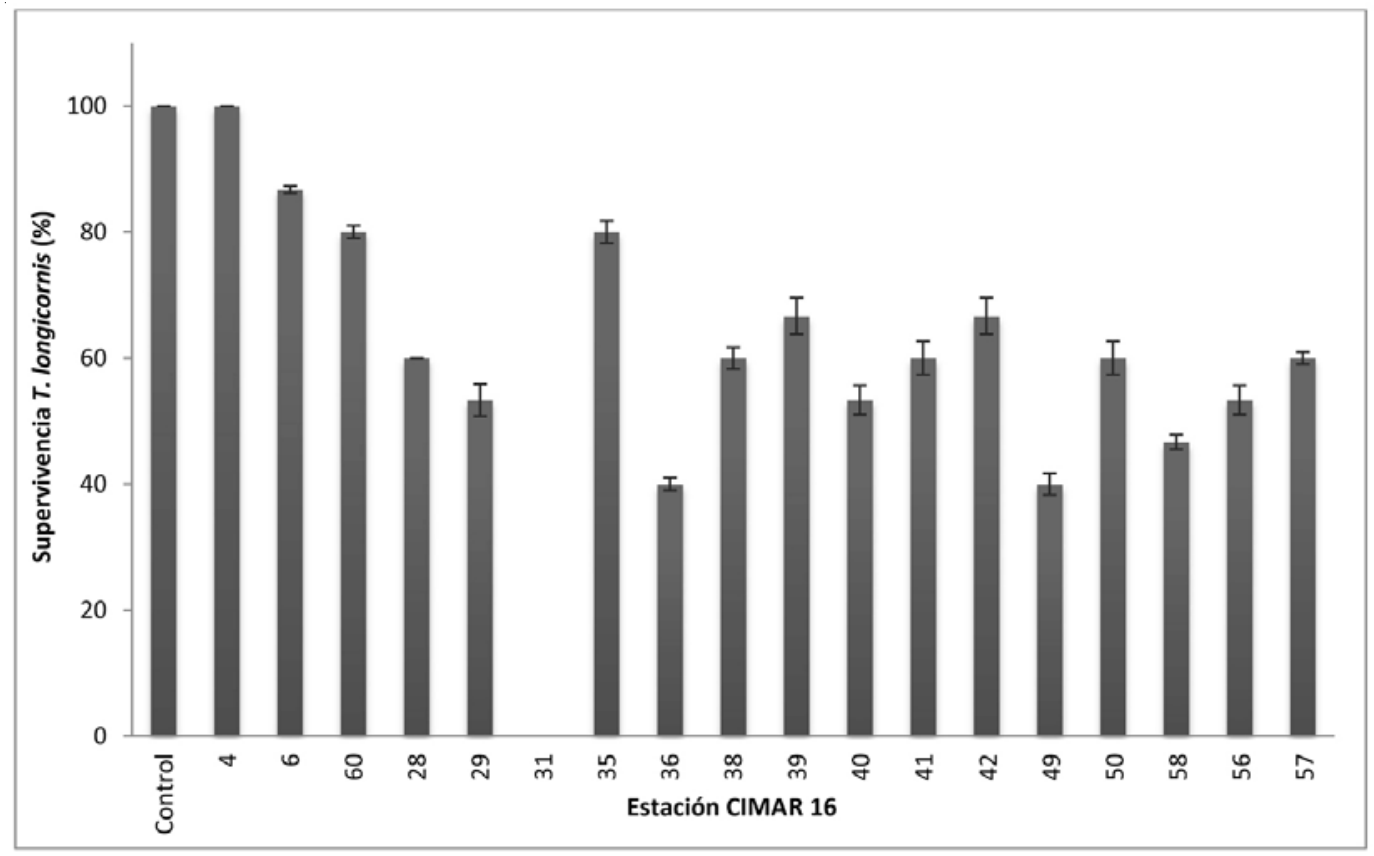

Figura 2. Porcentaje de supervivencia promedio ( \pm d.e) de $T$. longicornis en los ensayos con sedimentos analizados y grupo control. CIMAR 16 Fiordo, octubre-noviembre 2010 / Average survival rate ( \pm s.d.) of $T$. longicornis in sediments assays and control group. CIMAR 16 Fjord, October-November 2010

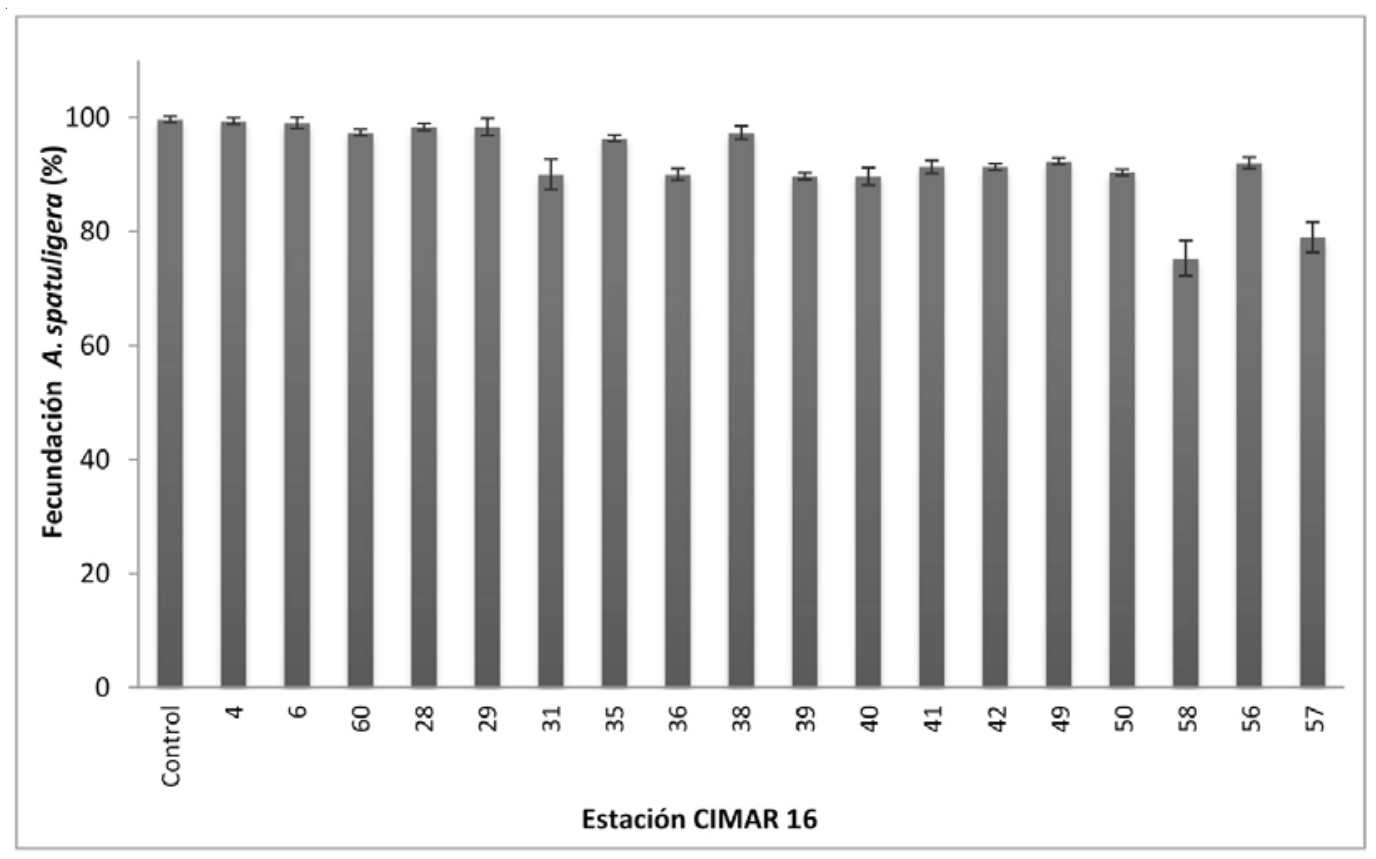

Figura 3. Gráfico de fecundación (\%) de gametos de $A$. spatuligera en los ensayos de toxicidad de los sedimentos del área estudiada (media \pm d.e). CIMAR 16 Fiordo, octubre-noviembre 2010 / Fertilization of $A$. spatuligera gametes (\%) in toxicity tests with sediment from the study area (mean \pm s.d). CIMAR 16 Fjord, October-November 2010 


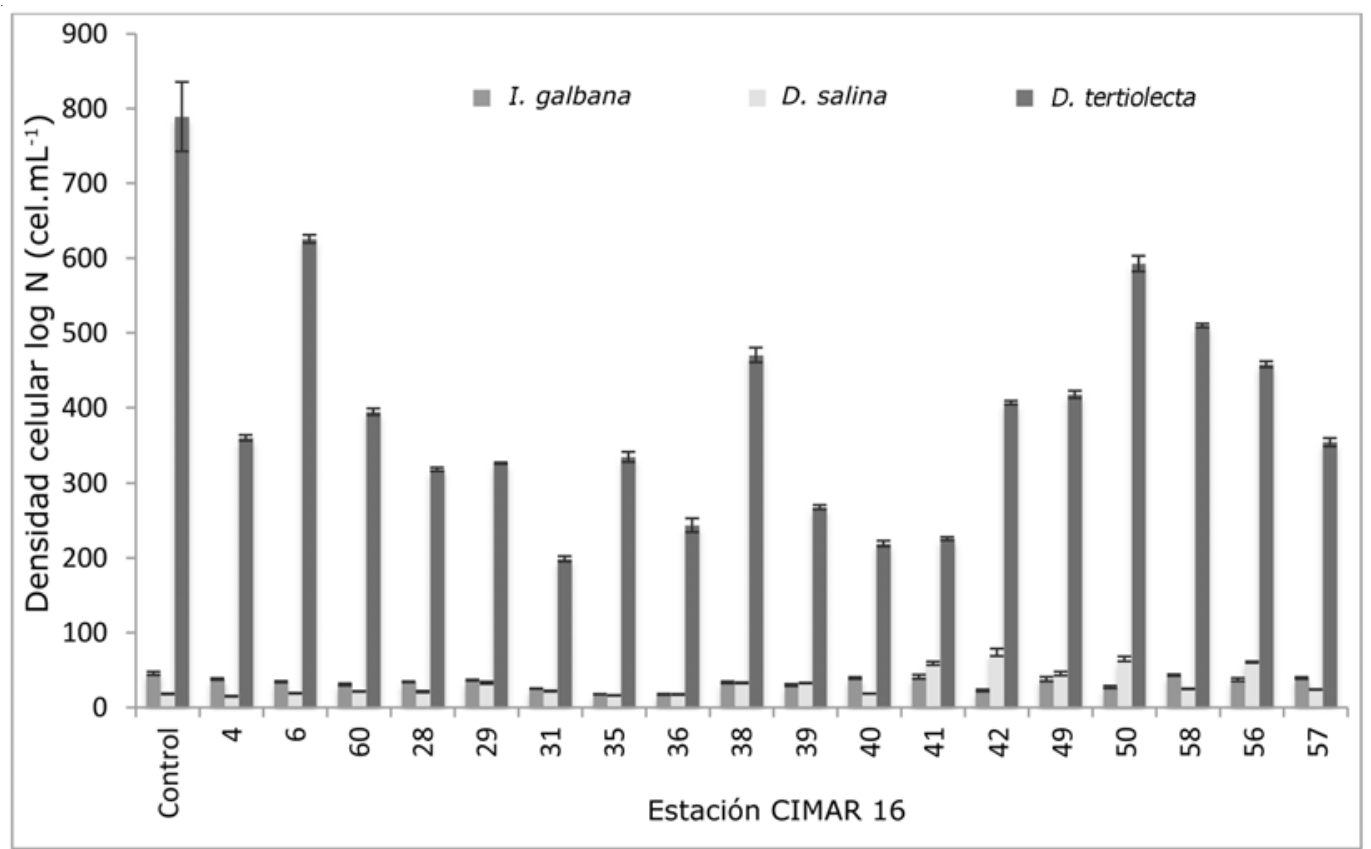

Figura 4. Crecimiento promedio (media \pm d.e) de I. galbana, $D$. salina y $D$. tertiolecta en los ensayos con los sedimentos de las áreas analizadas y grupo control. CIMAR 16 Fiordo, octubre-noviembre 2010 / Average growth (mean \pm s.d.) of I. galbana, D. salina, and D. tertiolecta in assays with sediments from areas analyzed and control group. Fjord CIMAR 16, October-November 2010

Los resultados del análisis de componentes principales permitieron observar cómo se agrupan las 3 microalgas de acuerdo a su crecimiento (Fig. 5). Las 2 primeras componentes representaron un $78,6 \%$ de la varianza total, con la mayor densidad celular, correspondiendo a las estaciones 50, 56, 49, 58, 38 y control. En especial, $I$. galbana y $D$. tertiolecta presentaron la mayor densidad en sus respectivos grupos control, la menor densidad celular de las microalgas se observó en la estación 31. El mayor crecimiento de $D$. salina se observó en los sedimentos de la estación 42.

El dendrograma usado como análisis exploratorio de semejanzas y/o diferencias en el resultado de los bioensayos con los sedimentos, muestra la agrupación de 4 conglomerados a una distancia de 2,65 unidades euclidianas (Fig. 6). Los extremos están marcados por el grupo control y la estación 31. Otro grupo estaría formado por las estaciones $41 \mathrm{y} 42$. El resto de las estaciones muestra un complejo agrupamiento con una similitud del $88 \%$.

\section{Discusión}

El análisis de los contenidos de materia orgánica en el sedimento de los canales y fiordos estudiados presentó en el $67 \%$ de las muestras valores cercanos al $5 \%$, lo cual corresponde con lo informado para el área i.e., 5,88 $\pm 1,88 \%$ por Silva y Ortiz (2002). Los valores medidos por sobre el $11 \%$ y bajo el $4 \%$ serían el resultado de la dinámica de las corrientes en las cuencas, i.e., bajas y altas, que generarían el depósito o el transporte del material en proceso de sedimentación, respectivamente. No se observaron asociaciones entre los resultados de las pruebas de calidad ambiental del sedimento, respecto de su contenido de materia orgánica total, ni con la profundidad en que fueron extraídas las muestras $(P>0,05)$.

Respecto de los ensayos de toxicidad en las pruebas de fecundación, se observaron porcentajes de fecundación significativamente menores que los controles $(P<0,05)$ en el $61,1 \%$ de las muestras. Aunque sólo en los sedimentos de las estaciones 58 y 57 se observaron porcentajes de fecundación menores al 90\%, lo que según 


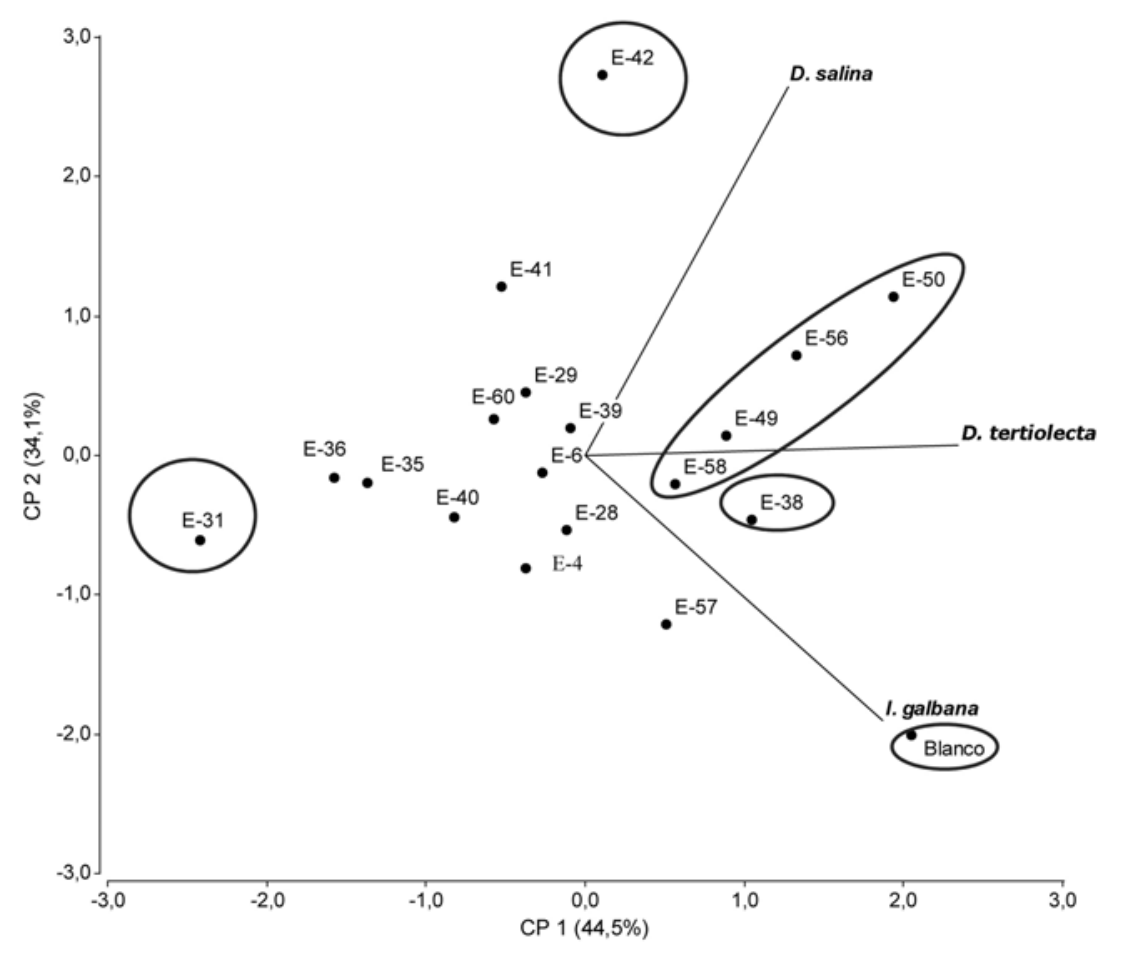

Figura 5. Análisis de componentes principales respecto de la densidad celular de las microalgas $D$. salina, D. tertiolecta y $I$. galbana medida en el elutriado de los sedimentos recolectados en el área de estudio y grupo control. CIMAR 16 fiordos / Principal component analysis of microalgal cell density $D$. salina, D. tertiolecta and I. galbana analyzed in the elutriated of sediments collected in the study area and control group. CIMAR 16 fjords

Aguirre-Martínez et al. (2009), puede considerarse distinto del comportamiento de los controles. Por otra parte, los ensayos de supervivencia con $T$. longicornis no arrojaron diferencias significativas respecto del grupo control ( $P>0,05)$, excepto en la estación 31 (Fig. 2), por lo que se puede aceptar que en general, las muestras de sedimentos analizadas no contienen residuos tóxicos, es decir, sustancias que alteren su respuesta respecto del grupo control.

En los ensayos con microalgas, la sensibilidad al $\mathrm{K}_{2} \mathrm{Cr}_{2} \mathrm{O}_{7}\left(\mathrm{CI}_{50}\right)$ fue semejante a la mostrada por las microalgas con que se analizó la calidad de los sedimentos en el sector norte de la Patagonia (Rudolph et al. 2007, 2009 y 2010), por lo que los resultados serían comparables. $\mathrm{Al}$ igual que lo observado en los ensayos del sector norte de la Patagonia (Rudolph et al. 2010), en el sector sur el crecimiento de D. tertiolecta fue mayor que lo mostrado por D. salina y I. galbana. Por otra parte, respuestas con mayor similitud se observaron entre I. galbana y $D$. tertiolecta, por lo que serían mejores indicadores en las pruebas de crecimiento celular que los ensayos con $D$. salina.

Las diferencias significativas $(P<0,05)$ observadas en la densidad celular de las estaciones en estudio, para las 3 microalgas al término del ensayo (i.e., 96 h) en relación a su grupo control, podrían indicar alguna diferencia/ alteración, que no necesariamente implica contaminación; ya que in situ se ha observado relativamente altos contenidos de oxígeno disuelto en la capa profunda i.e.,> $4 \mathrm{~mL} \mathrm{~L}^{-1}$ (Valdenegro \& Silva 2003). Estos autores lo atribuyen a la ventilación que genera el ingreso al interior de la zona de canales de Aguas Superficiales Subantárticas 
Promedio (Average linkage)

Distancia: (Euclidea)

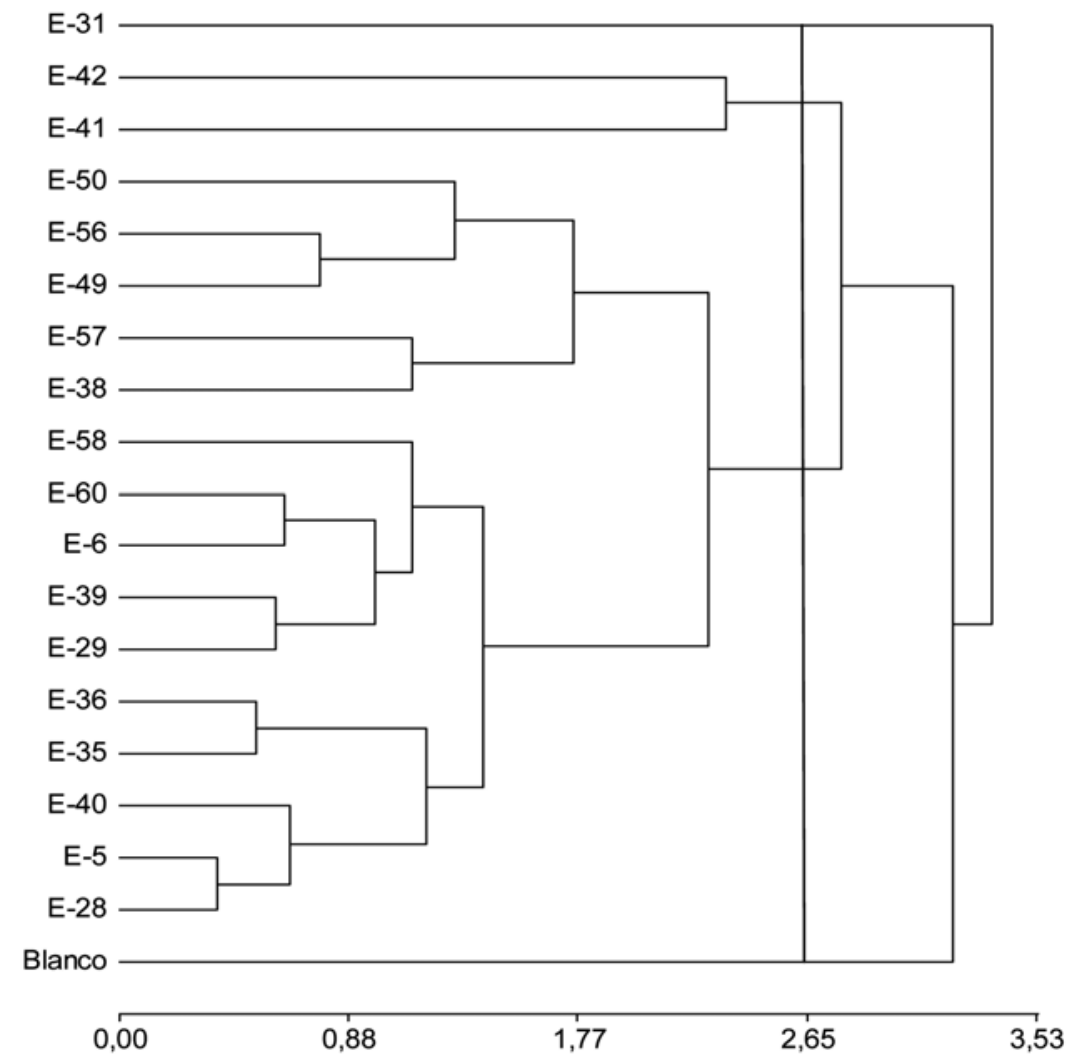

Figura 6. Dendrograma de la respuesta de crecimiento de las microalgas en los ensayos y el grupo control / Dendrogram of growth response of microalgae and control group

(0-100 m) bien oxigenadas, las que debido a su mayor densidad se hunden llenando las microcuencas.

El análisis de componentes principales y dendrograma permitió comprobar la sensibilidad de los ensayos utilizados en este estudio. Además, permitieron identificar las estaciones que se comportaron distintos de los grupos control, i.e., las estaciones 41 y 42 (sector sur) y estación 31 ubicada en la zona más externa (sector NW), posiblemente con la mayor influencia oceánica, generada por la cercanía de la costa y/o forma de la cuenca. Esta última i.e., estación 31, también presentó un comportamiento significativamente distinto en los ensayos de supervivencia con T. longicornis, lo que requiere de mayor análisis.

Se concluye que, debido a la escasa diferencia de los ensayos de fecundación, supervivencia y el crecimiento de las microalgas respecto de los controles se podría considerar a los sedimentos del área sur Patagónica con características prístinas.

\section{Agradecimientos}

Los resultados corresponden al Proyecto CONA-C16F 10-15. Los autores expresan sus agradecimientos al CONA, a la tripulación del AGOR Vidal Gormaz y a Emma Cascales por la toma de muestras durante el crucero.

\section{LITERATURA CITADA}

Aguirre-Martínez G, A Rudolph, R Ahumada, R Loyola \& V Medina. 2009. Toxicidad no específica en sedimentos portuarios, una aproximación al contenido de contaminantes críticos. Revista de Biología Marina y Oceanografía 44(3): 725-735. 
Ahumada R, A Rudolph \& S Contreras. 2002. Contenido de metales ( $\mathrm{Ba}, \mathrm{Cd}, \mathrm{Co}, \mathrm{Cu}, \mathrm{Ni}, \mathrm{Pb}, \mathrm{Sr}, \mathrm{V}$ y Zn) en los sedimentos marinos de la región patagónica $\left(52^{\circ}-56^{\circ} \mathrm{S}\right)$, Chile. Ciencia y Tecnología del Mar 25(2): 77-86.

Antezana T. 1999. Hydrographic feature of Magellan and Fueguian in land passages and adjacent subantarctic waters. Scientia Marina 63(suppl.1): 69-80.

Antezana T, A Giraldo \& M Hamamé. 2002. Clorofila y alimentación del zooplancton fraccionado por tamaño, en subcuencas del sistema de canales magallánicos y fueguinos durante la primavera de 1998. Ciencia y Tecnología del Mar 25(1): 109-130.

Borgel R. 1970-1971. Geomorfología de las regiones australes de Chile. Revista Geográfica de Chile, Terra Australis 20: 135-140.

Cifuentes A, J Silva, E Bay-Schmith \& A Larraín. 1998. Selección de cepas de microalgas para ser utilizadas en bioensayos de toxicidad. Gayana Oceanológica 61(1-2): 1-9.

Dinnel PA \& QJ Strober. 1985. Methodology and analysis of sea urchin embryo bioassays. Fisheries Research Institute, University of Washington, Seattle. Circular 85: 1-319.

Finney DJ. 1971. Probit analysis, 333 pp. Cambridge University Press, Cambridge.

Larrain A, E Soto, J Silva \& E Bay-Schmith. 1998. Sensibility of meiofaunal copepod Tisbe longicornis to $\mathrm{K}_{2} \mathrm{Cr}_{2} \mathrm{O}_{7}$ under varying temperature regimes. Bulletin of Environmental Contamination Toxicology 61: 391-396.

Luczak C, M Janquin \& A Kupka. 1997. Simple standard procedure for the routine determination of organic matter in marine sediment. Hydrobiologia 345: 87-94.

Mansilla A, M-E Ramírez, M Ávila, J Ojeda, JP Rodríguez \& S Rosenfeld. 2011. Macroalgas bentónicas de la Región de Magallanes y Antártica Chilena. Informes preliminares Crucero CIMAR 16 Fiordos: 209-227. Servicio Hidrográfico y Oceanográfico de la Armada de Chile, Valparaíso.

Pickard GL. 1971. Some physical oceanographic feature of inlets of Chile. Journal Fisheries Research Board of Canada 28: 1077-1106.

Rozzi R, F Massardo, A Mancilla, CB Anderson \& A Berghófer. 2007. La reserva de la biósfera para la conservación de la biodiversidad e implementación del desarrollo sustentable en el extremo austral de América. Anales del Instituto de la Patagonia, Ciencias Naturales 35: 55-62.

Rudolph A, G Aguirre, J Moscoso, N Silva \& R Ahumada. 2007. Sediment quality between Reloncaví Gulf and Corcovado Gulf (41.5-43ㅇ) based on toxicity tests. Investigaciones Marinas 35(2): 53-61.
Rudolph A, P Medina, C Urrutia \& R Ahumada. 2009. Ecotoxicological sediment evaluations in marine aquaculture areas of Chile. Environmental Monitoring and Assessment 155: 419-429.

Rudolph A, P Medina, V Novoa, R Ahumada \& I Cortés. 2010. Calidad ecotoxicológica de sedimentos en sectores del Mar Interior de Chiloé, Campaña CIMAR 12 Fiordo. Ciencia y Tecnología del Mar 33(1): 17-22.

Sievers H \& N Silva. 2008. Water masses and circulation in austral Chilean channels and fjords. In: Silva N \& S Palma (eds). Progress in the oceanographic knowledge of Chilean interior waters, from Puerto Montt to Cape Horn, pp. 5358. Comité Oceanográfico Nacional / Pontificia Universidad Católica de Valparaíso, Valparaíso.

Silva N \& P Ortiz. 2002. C y N su distribución y estereometría en sedimentos superficiales de la región sur de la zona de fiordos y canales australes de Chile, $52-56^{\circ} \mathrm{S}$ (Crucero CIMAR-FIORDO 3). Ciencia y Tecnología del Mar 25(1): 89-108.

Silva N \& S Palma. 2008. Progress in the oceanographic knowledge of Chilean interior waters, from Puerto Montt to Cape Horn, 161 pp. Comité Oceanográfico Nacional / Pontificia Universidad Católica de Valparaíso, Valparaíso.

Sobarzo M. 2009. La región de los fiordos de la zona sur de Chile: aspectos oceanográficos. En: Häusssermann V \& G Försterra (eds). Fauna marina bentónica de la Patagonia Chilena, pp. 53-60. Natura in Focus, Santiago.

USEPA. 1978. Selenastrum capricornutum Printz algal assay bottle test experimental design, application, and data interpretation protocol. Office of Research and Development. Environmental Research LaboratoryCorvallis, Oregon. EPA-600/9-78-018.<http://water . epa . gov/scitech/methods/cwa/wet/disk1_index.cfm>

USEPA. 1988. Short-term methods for estimating the chronic toxicity of effluents and receiving waters to marine and estuarine organisms. Office of Research and Development, U.S. Environmental Protection Agency. Washington D.C. 206460. EPA/600/4-87-028.<http://water.epa.gov/scitech/ methods/cwa/wet/disk1_index.cfm>

Valdenegro A \& N Silva. 2003. Caracterización física y química de la zona de canales y fiordos australes de Chile entre el Estrecho de Magallanes y Cabo de Hornos (CIMAR 3 Fiordo). Ciencia y Tecnología del Mar 26(2): 19-60.

Zúñiga M. 1999. Evaluación de la calidad acuática de bahía San Jorge a través de ensayos de toxicidad crónica con gametos del erizo de mar Arbacia spatuligera. Ciencia y Tecnología del Mar 22: 59-74.

Recibido 15 de abril de 2012 y aceptado 16 de diciembre 2013

Editor: Claudia Bustos D. 